\title{
The Relationship Between WAIS-R Abilities and Speed of Processing in a Word Identification Task
}

\author{
P. MCGeorge \\ J.R. CRAWFORD \\ S.W. KeLLY \\ University of Aberdeen
}

\begin{abstract}
Matched to the proportions found in the U.K. census data for a range of demographic variables (age, sex, and socioeconomic status) 123 participants were tested on the Wechsler Adult Intelligence Scale-Revised (WAIS-R) and a test of the minimum presentation time required to identify tachistoscopically presented words. The correlations between the sum of the scaled scores for Full, Verbal, and Performance subtests and the log of the identification measures were $-0.40,-0.22$, and -0.51 , respectively. These results are in line with those observed between the WAIS-R measures and standard visual inspection time (IT). Confirmatory factor analysis indicated that a three-factor model of intelligence with Verbal, Performance, and Attention/Concentration factors, and with the identification threshold loaded on the Performance factor alone, represented a better fit to the data than either a single general factor model or a two-factor model with Verbal and Performance factors. These results are in line with findings in the IT literature (Deary, 1993) that speed of information processing is significantly related to performance IQ but not to verbal IQ.
\end{abstract}

The idea that there is a relationship between intelligence and mental speed is an accepted part of our everyday language (Kline, 1991; Sternberg, 1985) and is a view that has a long and controversial history within psychology (Deary, 1986, 1994; Kline, 1991; Nettlebeck, 1987). It is a view that is closely identified with what Anderson (1992) referred to as low-level theories of intelligence (e.g., Eysenck, 1988). Low-level theories regard intelligence as a function of the speed and cfficiency with which the nervous system is capable of performing simple operations (Mackintosh, 1986). For example, Hunt (1978) proposed that individual differences in verbal intelligence could be understood largely in terms of

Thanks to Denis Parker for his comments on an earlier draft of this article and to Pat Chalmers for her help in collecting the data. Thanks also to Ian Deary, Arthur Jensen, and an anonymous reviewer for their helpful comments.

Preparation of this article was supported by a grant from the Economic and Social Research Council (R000234347).

Correspondence and requests for reprints should be sent to Peter McGeorge, Department of Psychology, University of Aberdeen, Old Aberdeen AB9 2UB, Scotland, U.K. 
differences among individuals in speed of access to lexical information in longterm memory. To test this Hunt and colleagues used the letter comparison task developed by Posner and Mitchel (1967). Participants are presented with pairs of lettcrs, for cxample, AA, Aa, or Ab, that could be similar either physically or in name. The task is to indicate as rapidly as possible whether the two letters are a match. In one condition participants indicate whether they are a physical match and in another whether they match in name. By subtracting the time to make comparisons concerning physical matches from the time taken to make comparisons concerning name matches Hunt and colleagues obtained a measure of speed of lexical access independent of speed of responding. The typical correlation between lexical access time and verbal ability was of the order -0.3 .

Currently, the most widely used of the "mental speed" paradigms employed to investigate the relationship between performance on intelligence tests and speed of information processing is inspection time, or IT (Vickers, Nettlebeck, and Wilson, 1972). In the standard inspection time task, using a tachistoscope, a participant is presented with two vertical lines, one longer than the other. These lines are followed by a backward mask, and the testee is asked to judge which of the two lines is the longer. The procedure is repeated with the presentation time for the lines varied in some systematic way until the person is just able to make the discrimination accurately (Mackintosh, 1986). In a comprehensive review and meta-analysis Kranzler and Jensen (1989) reported that the correlation between general IQ and IT was in the order of -0.30 prior to correction for various artifacts. The correlations between IT and performance IQ was in the order of -0.45 and between IT and verbal IQ, -0.18 . Following corrections for attenuation, sampling error, and range restriction these correlations increased to -0.54 , -0.69 , and -0.27 , respectively (Kranzler and Jensen, 1989).

The interpretation of the correlation between IT and IQ is not without controversy (Anderson, 1992; Kline, 1991). Anderson (1992) outlined two principal criticisms of the research into the relationship between IT and intelligence. The first is that many of the published studies have made use of participant samples that were restricted in range, for example, consisting only of undergraduate students, and/or were heterogeneous in respect of populations, for example, including normal and retarded adults in the same sample. The second criticism has concerned the idea that IT reflects a "pure" measure of mental speed. A number of studies have investigated the potential role of "strategic" influences on IT performance. For example, Egan (1994) investigated the use of apparent motion cues to aid IT discriminations. The results indicated that there was no difference in the IT IQ correlations for participants who did or did not report using apparent motion cues. Egan (1994) concluded that the use of the apparent motion cue strategy neither caused nor reduced the IT IQ correlation. Anderson (1992) argued that IT represents the purest of the mental speed measures.

Deary (1993) argued that there is little need to continue to replicate the finding 
that IT and IQ correlate and that it is more important to ask more detailed questions concerning the nature of the IT intelligence relationship. One such issue Deary identified is whether mental speed is more closely related to some forms of human mental ability than to others (Deary, 1993). For example, Nettlebeck (1987) argued that whereas the relationship between IT and performance IQ is relatively robust, the relationship between IT and verbal ability remains unresolved.

To address this issue Deary (1993) examined the relationship between performance on the standard visual IT task and WAIS-R IQ subtypes using both exploratory and confirmatory factor analysis. The results of the confirmatory factor analysis indicated that a two-factor model of intelligence with correlated Performance and Verbal factors and with IT loaded on the Performance factor only was a better fit to the data than a single general ability model. The fact that the models fit to the data would not have been improved by the addition of a nonzero loading of IT on the Verbal Ability factor suggests that IT is only weakly related to verbal ability (Deary, 1993).

Although the hypothesis that mental speed represents a basic, domain-independent, biological property of an individual's nervous system would suggest that the pattern observed in the study by Deary (1993) would hold for all tasks measuring mental speed, it does not rule out the possibility that alternative measures may show a different pattern of relationships. For example, the materials in the standard IT task are nonverbal, and so finding that performance in this task is unrelated to a Verbal Ability factor is, at one level, not surprising. Following from the suggestion by Hunt (1978) that differences in verbal intelligence can be understood by differences in speed of lexical access, we might expect that if verbal materials were used then participants with high verbal ability would have a lower access time than those with lower verbal ability. Using verbal materials might be expected to result in a nonzero path between the mental speed measure and the Verbal Ability factor-a different pattern to that reported by Deary (1993). A number of measures have been used to assess speed of lexical access (see Hunt, 1978). One direct way of assessing speed of lexical access is to measure the minimum duration for which a word must be presented in order for the testee to identify it correctly. This is the approach used in the study presented here. This study examines the relationship between psychometric measures of intelligence and threshold of identification for visually presented words using a large sample of adults matched against U.K. census data and screened for neurological, psychiatric, and sensory problems.

\section{PARTICIPANTS}

One hundred and twenty-three individuals took part in the study (60 males, 63 females). They were recruited from a wide range of sources and were free from 
TABI.F 1

Social Class Distribution in the Sample and the Adult U.K. Population (\%)

\begin{tabular}{lccccr}
\hline & \multicolumn{5}{c}{ Social Class } \\
\cline { 2 - 6 } & $\mathbf{1}$ & $\mathbf{2}$ & $\mathbf{3}$ & $\mathbf{4}$ & $\mathbf{5}$ \\
\hline Sample & 5 & 21 & 50 & 17 & 6 \\
General population & 5 & 23 & 48 & 18 & 6 \\
\hline
\end{tabular}

neurological, psychiatric, or sensory disorders. All of them received a small honorarium for their participation. Mean age was 44.9 years, $S D=17.14$, with a range of 18 to 77 . Participants were recruited so that the sample structure matched, as closely as possible, the structure of the U.K. census data concerning sex, age, and social-class distributions. The social-class distribution of the sample is presented in Table 1 along with the census-derived figures for the adult U.K. population. A goodness-of-fit Chi-square test revealed that the sample proportions did not differ significantly from the adult U.K. population, $\chi^{2}=0.31$, $d f=4, p>0.1$.

A similar procedure was adopted to examine the representativeness of the sample in terms of age distribution. Four age bands were formed (16-29, 30-44, $45-59,60+$ ). Each age band was approximately equivalent in size and represented approximately an equal proportion of the general population. The proportion of the same falling within each age band was calculated. Comparison of these proportions with the census-derived proportions (see Table 2) using a goodness-of-fit test revealed that they did not differ significantly, $\chi^{2}=0.75, d f=3$, $p>0.1$.

Finally, the sex distribution of the sample did not differ significantly from the census figures, $\chi^{2}=0.04, d f=1, p>0.1$.

TABLE 2

Age Distribution in the Sample and the Adult U.K. Population (\%)

\begin{tabular}{lccccc}
\hline & \multicolumn{4}{c}{ Age (years) } \\
\cline { 2 - 5 } & $\mathbf{1 6 - 2 9}$ & $\mathbf{3 0 - 4 4}$ & $\mathbf{4 5 - 5 9}$ & $\mathbf{6 0 +}$ \\
\hline Sample $(N=123)$ & 25 & 26 & 25 & 24 \\
General population & 27 & 25 & 22 & 26 \\
\hline
\end{tabular}




\section{METHODS}

\section{Determination of Word Identification Threshold}

For each participant a list of 50 words was used to determine the minimum time required to identify correctly one out of every three words presented using a standard three-field tachistoscope (Electronic Developments). All the words were common nouns between six and seven letters in length and of low to medium frequency of occurrence in English ( $\mathrm{M}=15.7$ occurrences/million, $S D=$ 8.5; Kucera and Francis, 1967). For each participant a starting point on the list was chosen at random.

Prior to the presentation of each word, testees focused on a central fixation point (field intensity $20 \%$ ), and the participant then initiated the presentation of the word. This was immediately followed by a 1-sec backward mask composed of three rows of ampersands (field intensity 60\%). The initial presentation time was for $110 \mathrm{msec}$ (field intensity 40\%). Following each correct identification the presentation time was decreased by $20 \mathrm{msec}$ down to $50 \mathrm{msec}$ and by decrements of $10 \mathrm{msec}$ below $50 \mathrm{msec}$. When the participant gave three incorrect responses in succession, the stimulus duration was increased to the halfway point between the duration at that time and the duration at the last correct response. This procedure was repeated until participants responded correctly on one out of three consecutive trials, averaged over six trials. The stimulus presentation time was recorded as the participant's threshold of identification. This criterion differs from the one traditionally used in visual inspection time studies and was chosen primarily to fit the requirements of subsequent studies of repetition priming in which all testees later participated. The criterion was chosen to allow sufficient degrees of freedom in later studies for priming effects to significantly increase the number of correct identifications.

\section{WAIS-R (Wechsler, 1981)}

A full-length WAIS-R was administered and scored according to standard procedures.

\section{RESULTS}

The mean full-scale IQ for the sample was $106.9(S D=13.2)$, and the mean verbal and performance IQs were $107.0(S D=13.7)$ and $105.4(S D=12.6)$ respectively. The mean threshold of identification for the verbal materials was $37.5 \mathrm{msec}(S D=17.7)$. The threshold values showed a slight negative skew and leptokurtosis. A logarithmic transformation produced a near normal distribution.

Table 3 shows the correlations between threshold values and WAIS-R IQs. The full correlations matrix for the threshold values and the WAIS-R subtests are presented in Appendix A. 


\section{TABLE 3}

Correlations Between WAIS-RIQ Measures and the Raw and Transformed Threshold Values

\begin{tabular}{lcc}
\hline & $\begin{array}{c}\text { Raw Threshold Scores } \\
(\mathbf{m s e c})\end{array}$ & Log-Transformed Threshold Scores \\
\hline Full-scale IQ & -.09 & -.09 \\
Verbal IQ & -.11 & -.12 \\
Performance IQ & -.06 & -.06 \\
\hline \multicolumn{2}{l}{$N=123}$. & \\
${ }^{*} p<0.05 .{ }^{* *} p<0.01 .{ }^{* * *} p<0.001$. &
\end{tabular}

The correlations between threshold values and full-scale, performance, and verbal IQs were not significant. However, using IQ summary statistics in samples where participants may vary widely in age can result in a considerable loss of information. It could be argued that in examining the relationship between the threshold measure and the WAIS-R abilities, either both measures should be uncorrected for age or both measures should be corrected for age. The threshold measure is uncorrected for age. The correlations between the sum of the scaled subtest scores (uncorrected for age), the individual subtest scores, and both the raw and transformed threshold values are presented in Table 4 .

The correlations between threshold and the sum of the scaled scores for the

\section{TABLE 4}

Correlations Between the Sum of Scaled Scores, Scaled Subtest Scores, and Between These and the Raw and Transformed Threshold Values

\begin{tabular}{lcc}
\hline & $\begin{array}{c}\text { Raw Threshold Scores } \\
(\mathbf{m s e c})\end{array}$ & $\begin{array}{c}\text { Log-Transformed Threshold } \\
\text { Scores }\end{array}$ \\
\hline Sum of scaled scores & $-.37^{* *}$ & $-.40^{* *}$ \\
Sum of verbal subrest scaled scores & $-.21^{*}$ & $-.22^{* *}$ \\
Sum of performance subtest scales scores & $-.47^{*+*}$ & $-.51^{* * * * *}$ \\
Information & -.13 & -.15 \\
Digit span & -.12 & -.09 \\
Vocabulary & -.09 & -.09 \\
Arithmetic & $-.24^{* *}$ & -.22 \\
Comprehension & -.11 & -.14 \\
Similarities & $.30^{* *}$ & $.35^{* * *}$ \\
Picture completion & $-.26^{* *}$ & $-.27^{* *}$ \\
Picture arrangement & $-.33^{* *}$ & $-.37^{* * *}$ \\
Block design & $-.35^{* *}$ & $-.38^{* *}$ \\
Object assembly & $-.39^{* *}$ & $-.44^{* *}$ \\
Digit symbol & $-.41^{*+}$ & $-.44^{* * *}$ \\
\hline
\end{tabular}

$$
\begin{aligned}
& N=123 . \\
& { }^{*} p<0.05 .{ }^{* *} p<0.01 .{ }^{* * * *} p<0.001 .
\end{aligned}
$$


verbal and performance measures were significant. Using Williams's (1959) test, the correlation between threshold of identification and the sum of the scaled scores for the performance subtests was significantly higher than the correlation between threshold of identification and performance IQ, $t(120)=9.18, p>$ 0.001 . Similarly, the correlation of threshold of identification with the sum of the verbal subtests was significantly higher than the correlation with verbal IQ $t(120)$ $=4.21, p>0.001$.

The threshold measure had a significantly higher correlation with the sum of performance subtests than the sum of verbal subtests, $t(120)=3.73, p>0.001$.

\section{Confirmatory Factor Analysis}

A confirmatory factor analysis was conducted on the transformed threshold scores and WAIS-R subtest scores using the EQS program (Bentler, 1989).

Factor analytic studies of the 11 subtests of the WAIS-R have consistently indicated that it can be characterized by a Global factor $(g)$, a Verbal Ability/Comprehension factor, a Perceptual Organization factor, and a Freedom from Distractability or Attention/Concentration factor (Kaufman, 1990). Based on this, three models were constructed to try and account for the pattern of covariances between tests (see Figure 1). First, a single-factor model (MI) in which all 11 subtests of the WAIS-R and the threshold measure were caused by a single underlying factor common to all tests-general intelligence $(\mathrm{g})$, and variance specific to each test. Second, a two-factor model was constructed (M2). Verbal subtests of the WAIS-R were assumed to have nonzero loadings on the first factor and zero loadings on the second; performance subtests were assumed to have nonzero loadings on the second factor and zero loadings on the first. The threshold measure had a zero loading on the verbal factor and nonzero loading on the perceptual factor. Finally, a three-factor model was constructed (M3). All the performance subtests were assumed to have nonzero loadings on the second factor and zero loadings on the other factors. All verbal subtests except digit span and arithmetic were assumed to have nonzero loadings on the first factor and zero loadings on the other factors. Digit span and arithmetic were assumed to have nonzero loadings on the third factor and zero loading on all other factors. The threshold measure was assumed to have a nonzero loading on the Perceptual Organization factor and zero loadings on the other factors. In addition, in the two- and three-factor model, all factors were assumed to be correlated and so implicitly contain a second-order factor, general intelligence $(g)$.

Fit indices for each of the models are presented in Table 5. A sequential comparison of the differences in goodness-of-fit for the three models was conducted. Following guidelines specified by Bentler and Bonnet (1980) differences between the Chi-square statistic and the degrees of freedom associated with each model were calculated and then evaluated for statistical significance. These comparisons allow the extent to which competing models are better fits of the data to be evaluated. 
(a) M1: Single Factor Model

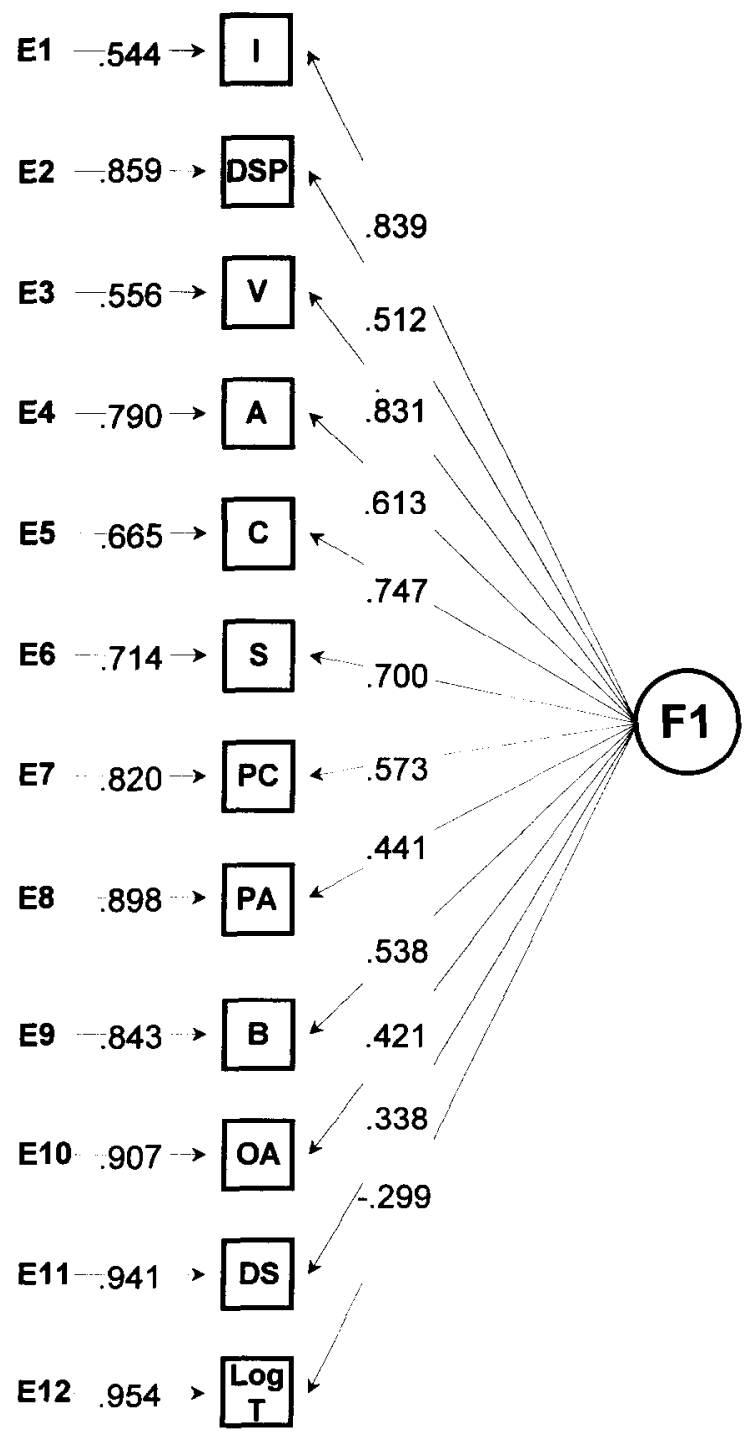

Figure 1. Single-factor general ability model (M1), (b) two-factor model (M2) with correlated Verbal Ability and Perceptual Organization factors, (c) three-factor model (M3) with correlated Verbal, Perceptual Organization, and Concentration factors. I, Information; V, Vocabulary; C, Comprehension; S, Similarities; PC, Picture Completion; PA, Picture Arrangement; B, Blocks Design; OA, Object Assembly; DS, Digit Symbol; DSP, Digit Span; A, Arithmetic. 
Figure 1. (Continued)

(b) M2: 2 Factor Model

$$
\begin{aligned}
& E 1-.487 \rightarrow \square \\
& E 2-.862 \rightarrow \text { DSP } \quad .873 \\
& \mathrm{E3}-.442 \rightarrow \mathrm{V}{ }^{.507} \\
& \text { E4 }-.819 \rightarrow \mathrm{A} \times{ }_{.574}^{.897} \\
& \text { E5 }-.616 \rightarrow \mathrm{C} *{ }_{.788} \\
& E 6-.772 \rightarrow \mathrm{s} \leftarrow .635 \ldots \mathrm{F1}
\end{aligned}
$$

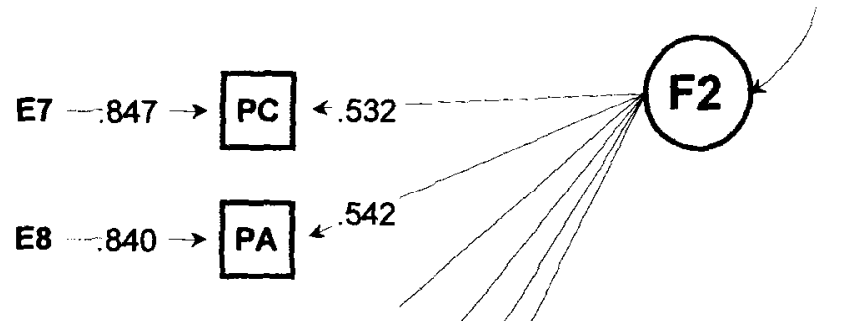

$$
\begin{aligned}
& \text { E9 }-.559 \rightarrow \mathrm{B} *{ }_{.806}^{.830} \\
& \mathrm{E10}-.592 \rightarrow \mathrm{OA} \times .570 \\
& E 11-822 \rightarrow \text { DS }-530 \\
& \text { E12 } 848 \rightarrow\left[\begin{array}{c}
\text { Log } \\
T
\end{array}\right.
\end{aligned}
$$


Figure 1. (Continued)

\section{(c) M3: 3 Factor Model}

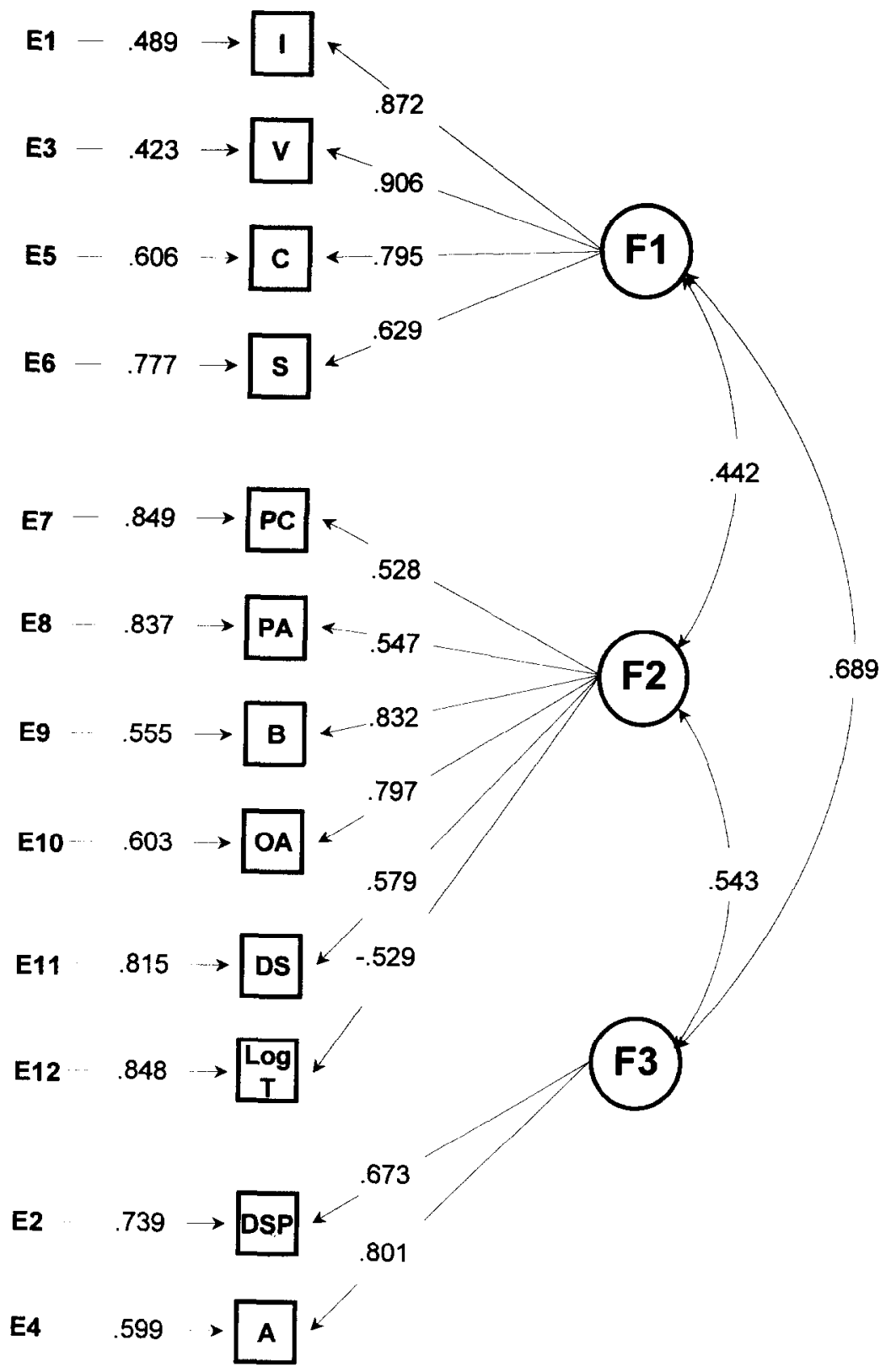


TABLE 5

Model Fit Statistics

\begin{tabular}{lcccc}
\hline & $\begin{array}{c}\text { Bentler-Bonnet } \\
\text { Normed } \\
\text { Fit Index }\end{array}$ & $\begin{array}{c}\text { Bentler-Bonnet } \\
\text { Nonnormed } \\
\text { Fit Index }\end{array}$ & $\begin{array}{c}\text { Comparative } \\
\text { Fit Index }\end{array}$ & $\mathbf{X}^{2}(d f)$ \\
\hline M1-general intelligence & 0.611 & 0.577 & 0.654 & $\begin{array}{c}285.4(54) \\
p<0.001\end{array}$ \\
& 0.782 & 0.801 & 0.840 & $\begin{array}{c}159.9(53) \\
p<0.001\end{array}$ \\
$\begin{array}{l}\text { M2-verbal and performance } \\
\text { factors }\end{array}$ & 0.816 & 0.837 & 0.874 & $\begin{array}{c}135.4(51) \\
p<0.001\end{array}$ \\
$\begin{array}{l}\text { M3-verbal, performance, } \\
\text { concentration }\end{array}$ & & & & \\
\hline
\end{tabular}

Comparison between the general intelligence model (M1) and the two-factor model (M2) yielded a Chi-square difference statistic of 125.5, $d f=1, p<0.01$. Comparison of the two-factor model with the three-factor model yielded a Chisquare statistic of $24.5, d f=2, p<0.001$. The results indicate that the twofactor model represents a significant improvement in the fit to the data over the single-factor model and that the three-factor model represents a further, significant, improvement in fit over the two-factor model (a Schmid-Leiman orthogonalization of the model three-factor matrix is presented in Appendix B).

Bentler (1989) suggested that the fit indices of a well-fitting model should be greater than 0.9. None of the models evaluated in this study achieved values in this range. To test which parameters would need to be altered in order to significantly improve the model's fit, Lagrange multiplier (LM) and Wald tests (Bentler, 1989) were conducted for the three-factor model. The LM Test is used to examine where parameters that are currently fixed with zero values could be nonzero. The Wald Test is used to examine whether any of the parameters of the model that currently have nonzero values could be set to zero without any significant loss in the model's overall fit to the data. The results of the Wald Test indicated that there were no parameters in the model currently with nonzero values that could be set to zero. The results of the LM Test indicated that there were a number of pathways associated with the WAIS-R subtests that if allowed nonzero parameters would lead to an improvement in the overall fit of the model. Improvements in the fit between the model and the data could be achieved with nonzero path coefficients for the Similarities subtest and the Perceptual Organization factor, the Picture Completion subtest and the Verbal Ability factor, and the Object Assembly Test and the Attention/Concentration factor. Neither the Wald nor the LM tests indicated changes to the relationship between the threshold measure and the latent factors. 


\section{DISCUSSION}

The pattern of correlations observed between the minimum time that a word must be presented in order for it to be identified correctly (threshold of identification) and the various WAIS-R derived intelligence measures replicate the pattern reported for the correlations between IT and the WAIS-R measures (e.g., Deary, 1993; Kranzler and Jensen, 1989). The correlations between threshold of identification and the sum of the scaled scores for the full scale, and for the verbal and performance subtests were significant with the correlation between the threshold measure and the sum of performance subtests being significantly higher than its correlation with the sum of verbal subtests.

The pattern of correlations observed between the threshold measures and the age-corrected mental scores (IQ) is very different to that observed for the ageuncorrected mental scores (sum of scaled scores). In the case of the age-corrected scores none of the correlations with the threshold measures are significant, whereas for the age-uncorrected scores all the correlations with the threshold measures are significant. This difference in the results observed for age-corrected and age-uncorrected scores highlights the problems of using age-corrected scores in samples with large age ranges and in which some of the other measures being analyzed (e.g., threshold) are not age corrected.

The results of the confirmatory factor analysis (CFA) replicate and extend previous findings for the standard visual IT task (Deary, 1993). In the sludy by Deary (1993) the results of the CFA indicated that a two-factor model with correlated Performance and Verbal Ability factors, and with IT loaded on the Performance factor alone, was a better fit to the data than a single general ability model. Factor analytic studies of the WAIS-R have consistently indicated that in addition to Verbal and Performance factors it can be characterized by a Freedom from Distractibility or Attention/Concentration factor (Kaufman, 1990). The two most consistent indicators of the Attention factor are the digit span and arithmetic subtests. Deary (1993) used only 9 of the 11 WAIS-R subtests in his study and so could not consider a three-factor model.

The results of this study indicated that a three-factor model with correlated Verbal Ability, Performance, and Attention factors was a better fit to the data than either a single general ability model or a two-factor model. These results are in line with similar findings by O'Grady (1983). The three-factor model had zero paths between the threshold measure and the Verbal Ability and Attention factors. The LM Test indicated that the model's fit would not have been significantly improved by allowing these paths to be nonzero. The interpretation of the relationships between the mental speed task used in this study and the WAIS-R subtypes does not differ from that of the standard visual IT task (e.g., Deary, 1993). As with IT, the threshold measure is closely related to the Performance factor alone. The Verbal Ability factor and the Attention factor play little role except through their correlations with the Performance factor. This is in spite of 
the verbal nature of the materials in the threshold task and the superficial appearance of a strong attentional requirement in the task.

It has been suggested that, as a rule of thumb, the value of the fit indices in a well-fitting model should be greater than 0.9 (Bentler, 1989). None of the models evaluated in the study presented here achieved this level of fit. However, the results of the Wald and LM tests indicated that the model's fit to the data could be significantly improved by the addition of a number of nonzcro paths between the various WAIS-R subtests and latent factors. However, since none of these additional pathways involved the threshold measure, they would not require a reinterpretation of the threshold-WAIS-R subtypes relationships. If the additional pathways for the WAIS-R subtests are included in the model, then the fit indices increase to beyond the 0.9 level indicating a well-fitting model.

In the standard IT task, participants are required to make discriminations between nonverbal materials, whereas in this study they were required to identify briefly presented words. The aim of using words as the stimuli in the mental speed task was to test the prediction that the use of verbal materials would result in an advantage for subjects of high verbal ability. No such advantage was found; based on the results of the CFA, verbal ability had no appreciable direct effect on the threshold of identification. The fact that both types of materials result in a similar pattern of relationships to the standard psychometric measures of intelligence would appear to argue for what Anderson (1992) referred to as a lowlevel, domain-independent view of the role of mental speed in cognitive ability.

\section{REFERENCES}

Anderson, M. (1992). Intelligence and development: A cognitive theory. Oxford: Blackwell.

Bentler, P. (1980). Multivariate analysis with latent variables: Causal modeling. Annual Review of Psychology, 31, 419-456.

Bentler, P. (1989). EQS structural equations program manual. Los Angeles, CA: BMDP Statistical Software.

Bentler, P., \& Bonnet, D.G. (1980). Significance tests and goodncss-of-fit in the analysis of covariance structures. Psychological Bulletin, 88, 588-606.

Deary, I.J. (1986). Inspection time: Discovery or rediscovery. Personality and Individual Differences, $7,625-631$.

Deary, I.J. (1993). Inspection time and WAIS-R IQ subtypes: A confirmatory factor analysis study. Intelligence, 17, 223-236.

Deary, I.J. (1994). Sensory discrimination and intelligence: Post-mortem or resurrection? American Journal of Psychology, 107, 95-115.

Egan, V. (1994). Intelligence, inspection time and cognitive strategies. British Journal of Psychology, 85, 305-315.

Eysenck, H.J. (1988). The concept of "intelligence": Useful or useless? Intelligence, 12, 1-16.

Hunt, E. (1978). Mechanics of verbal ability. Psychological Review, 85, 109-130.

Kaufman, A.D. (1990). Assessing adolescent and adult intelligence. London: Allyn and Bacon.

Kline, P. (1991). Intelligence: A psychometric view. London: Routledge.

Kucera, M., \& Francis, W. (1967). Computational analysis of present-day American English. Providence: Brown University Press. 
Kranzler, J.H. \& Jensen, A.R. (1989). Inspection time and intelligence: A meta-analysis. Intelligence, 13, 329-347.

Mackintosh, NJ. (1986). The biology of intelligence. British Journal of Psychology, 77, 1-18.

Nettlebeck, T. (1987). Inspection time and intelligence. In P. Vernon (Ed.), Speed of information processing and intelligence. Norwood, NJ: Ablex.

O'Grady, K.E. (1983). A confirmatory maximum likelihood factor analysis of the WAIS-R. Journal of Consulting and Clinical Psychology, 5I, 826-831.

Posner M., \& Mitchel, R. (1967). Chronometric analysis of classification. Psychologiral Review, 74, 392-409.

Silverstein, A.B. (1985). Cluster analysis of the Wechsler Adult Intelligence Scale-Revised. Journal of Clinical Psychology, 4l, 98-100.

Sternberg, R.J. (1985). Beyond IQ: A triarchic theory of human intelligence. London: Cambridge University Press.

Vickers, D., Nettlebeck, T., \& Wilson, R.J. (1972). Perceptual indices of performance: The measurement of "inspection time" and "noise" in the visual system. Perception, 1, 263-295.

Wechsler, D. (1981). Manual for the Wechsler Adult Intelligence Scale-Revised. New York: Psychological Corporation.

Williams, E.J. (1959). The comparison of regression variables. Journal of the Royal Statistical Society, Series B, 21, 396-399. 